\title{
Akademisierung der Pflege 3.0
}

Online publiziert: 17. Mai 2012

(C) Springer-Verlag 2012

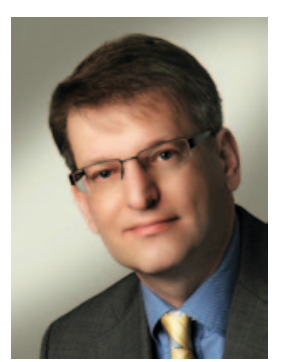

Jörg Klewer

Die Diskussion über die Novellierungen der Beruferichtlinie 2005/36/EU sowie des Krankenpflegegesetzes in Deutschland wirft die Frage auf, inwieweit HeilberufeSCIENCE auf diese Entwicklungen ausgerichtet ist. Wenngleich HeilberufeSCIENCE eine internationale Ausrichtung aufweist, so lässt sich bei dem deutschsprachigen inhaltlichen Schwerpunkt die Situation in Deutschland nicht ignorieren. Im Sinne der Systematik des Nummerierens von Entwicklungsstadien zeigt sich, dass HeilberufeSCIENCE von der inhaltlichen Ausrichtung her dem Level 2.0 der Akademisierung der Pflege (und letztlich auch der weiteren Gesundheitsfachberufe) entspricht. Was ist damit gemeint?

Für den deutschsprachigen Raum umfasst Akademisierung der Pflege 2.0 den Zeitraum seit ca. 1990, der mit der Gründung von Studiengängen in den Schwerpunkten Pflegewissenschaft, Pflegemanagement und Pflegepädagogik einherging und sich später auf weitere Gesundheitsfachberufe erweitert hat. (Akademisierung der Pflege 1.0 wäre demnach die Phase vor 1990, in der Pflegekräfte und andere Angehörige der Gesundheitsfachberufe nach Ausbildung und ggf. beruflicher Tätigkeit ein Studium absolviert haben, das nur einen indirekten Bezug zur vorherigen Berufstätigkeit hatte.)
Die inzwischen weit verbreitete Akademisierung in den Gesundheitsfachberufen und die Diskussion um Delegation und Substitution ärztlicher Leistungen erfordert die Diskussion über ein neues Level der Akademisierung: die Ausbildung von therapeutischen Experten, die eigenverantwortlich handeln und entscheiden dürfen - vergleichbar zum amerikanischen Advanced Nurse Practitioner. Mit dieser Akademisierung der Pflege 3.0 sollte keineswegs eine Konkurrenzprofession gegenüber der Medizin geschaffen werden, sondern letztlich ein Miteinander mit dem Ziel einer optimalen Patientenversorgung.

Um diese Diskussion zu vertiefen, sind u. a. die Themen Akademisierung und Übertragung ärztlicher Tätigkeiten Schwerpunkte des 10. Gesundheitspflege-Kongresses am 26. und 27. Oktober 2012 in Hamburg, zu dem ich Sie gern einladen möchte. Ferner möchte ich die Gelegenheit nutzen und Sie auf das „Call for Abstracts“ für das 3. HeilberufeSCIENCE-Symposium im Rahmen dieses Kongresses hinzuweisen. Die Abstracts der Poster und Vorträge werden auch dieses Jahr zitierfähig in einem HeilberufeSCIENCE Sonderheft veröffentlicht. Wenn Sie sich inhaltlich am 3. HeilberufeSCIENCE-Symposium mit einem Poster und ggf. einem Vortrag beteiligen möchten, würde es mich freuen, wenn Sie bis zum 31.07.2012 ein Abstract einreichen (www.heilberufe-kongresse.de).

In der nun veröffentlichten Ausgabe von HeilberufeSCIENCE bieten wir Ihnen fünf Beiträge und eine wissenschaftliche Kurzmitteilung. Die Ausgabe startet mit einem Artikel von Heidenreich und Kuhnke-Wagner zum Zusammenhang zwischen Aggression der Bewohner und Arbeitsfähigkeit des Personals in geriatrischen, nichtpsychiatrischen und nichtklinischen Einrichtungen. Daran schließt sich der Beitrag von Weiskopf et al. mit den Ergebnissen einer retrospektiven Dokumentenanalyse zum zunehmenden Phänomen der Scheidung im Alter nach langjähriger Ehe an. 
Die Studie von Ullrich widmet sich ebenfalls dem Thema der älter werdenden Bevölkerung, indem Internetauftritte und Monitore von Fahrkartenautomaten von öffentlichen Verkehrsgesellschaften hinsichtlich der seniorengerechten Gestaltung analysiert wurden. Der dritte Artikel, der sich mit der älteren Bevölkerung befasst, ist die Studie von Pallauf et al., die sich aus gerontologischer Sicht mit dem Skifahren im Alter auseinandersetzt. Der abschließende Artikel von Buttinger und Stummer präsentiert Ergebnisse einer Pilotstudie zur integrierten Schlaganfallversorgung in Oberösterreich. Den Abschluss bildet die wissenschaftliche Kurzmitteilung von Lindeman et al. mit einer Darstellung der Ergebnisse einer Querschnittsstudie zum Sexualverhalten von Auszubildenden in Gesundheitsfachberufen, vorrangig in der Krankenpflege.
An dieser Stelle möchte ich Sie wieder einladen, diese fünf Artikel und den wissenschaftlichen Kurzbeitrag dieser Ausgabe von HeilberufeSCIENCE kritisch zu lesen und in den wissenschaftlichen Dialog zur Akademisierung (der Pflege) 3.0 einzutreten - und natürlich würde es mich freuen, wenn Sie ein Abstract für das 3. HeilberufeSCIENCESymposium einreichen würden.

Ihr

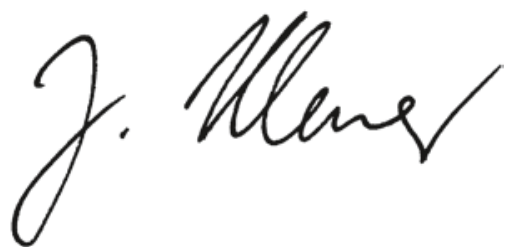

